\title{
Aproximaciones metateóricas sobre el constructo de los sistemas contables*
}

\section{Héctor Darío Betancur, Abel María Cano Morales}

En este artículo, se presentará el análisis del desarrollo disciplinar contable, partiendo del hecho del deber ser, la forma de vislumbrar y reinterpretar el concepto de sistema contable en el marco de las dinámicas socioeconómicas emergentes. Asimismo, se realizará un recorrido a través de la reconstrucción de su cuerpo teórico y, por esta vía, se propondrán alternativas de comprensión y entendimiento del hacer contable al interior de las organizaciones.

En el desarrollo de esta investigación, se forjó una reconstrucción de los problemas que comparten las teorías que abordan la noción de sistema contable, y, sobre todo, de los lenguajes formales que se usan en su descripción semántica y sintáctica. Finalmente, es preciso mencionar que esta es una investigación de corte analítico, que hace uso de la revisión documental para que, en un primer momento, se produzca una selección y sistematización, y, en un segundo, una esquematización a través del lenguaje semiformalizado. Lo anterior se realiza con la finalidad de proponer una dimensión objetiva y subjetiva, elaborada por la comunidad contable sobre la categoría sistema contable. De esta manera, es posible obtener información que allane el camino para su reconstrucción.

Palabras clave: Sistema contable, dimensión conceptual, estabilidad estructural, teoría disciplinar

Código JEL: M41, M42, M49

\section{Meta-theoretical approaches to the construction of accounting systems}

This article analyses the disciplinary accounting development, based on the way of glimpsing and reinterpreting the concept of accounting system within the framework of emerging socio-economic dynamics. Drawing from a journey through the reconstruction of its theoretical body, we will propose alternatives for understanding the accounting process within organizations. The development of this research has forged a reconstruction of the problems shared by the theories focus on the notion of accounting system and, especially, the formal languages used in its semantic and syntactic description. Finally, it is worth mentioning that this analytical research uses documentary review, on a first level, selecting and systematizing, and, on a second level, schematizing the information through semi-formalized language.

\footnotetext{
Este artículo es uno de los productos, resultado de la ejecución de la investigación sobre el análisis sistémico-constructivista de la noción de sistema contable como equivalente funcional de un mismo problema conceptual de referencia planteamientos filosóficos de la formación contable en Latinoamérica, en el marco del Doctorado en Ciencias Contables, y hace complemento con la investigación «Proceso de formación de contadores públicos que puedan atender las necesidades de la globalización en Latinoamérica».
} 
Thus, the article proposes an objective and subjective dimension, prepared by the accounting community on the accounting system category, allowing to obtain information that paves the way for its reconstruction.

Keywords: Accounting System, conceptual dimension, structural stability, disciplinary theory

JEL code: M41, M42, M49

\section{Abordagens meta-teóricas para a construção de sistemas de contabilidade}

Nesta seção, será feita uma apresentação sobre a análise do desenvolvimento contábil disciplinar, com base no fato do que deveria ser, a maneira de vislumbrar e reinterpretar o conceito de sistema contábil dentro da estrutura da dinâmica socioeconômica emergente. Da mesma forma, uma jornada será feita através da reconstrução de seu corpo teórico e, assim, propor alternativas para a compreensão e compreensão do processo contábil dentro das organizaçôes. No desenvolvimento desta pesquisa, uma reconstrução dos problemas compartilhados pelas teorias que falam sobre a noção de sistema contábil e especialmente as linguagens formais utilizadas em sua descrição semântica e sintática foi forjada. Por fim, é necessário mencionar que se trata de uma pesquisa analítica, que faz uso da revisão documental para que ocorra, inicialmente, uma seleção e sistematização, e uma segunda, uma esquematização através da linguagem semi-formalizada. O acima exposto, com o objetivo de propor uma dimensão objetiva e subjetiva, elaborada pela comunidade contábil na categoria de sistema contábil, permite obter informaçóes que possibilitem a sua reconstruçáo.

Palavras-chave: Sistema Contábil, dimensão conceitual, estabilidade estrutural, teoria disciplinar

Código JEL: M41, M42, M49.

\section{Introducción}

Una de las principales pretensiones de este trabajo es realizar una reconstrucción teórica de la noción que se ha elaborado sobre sistema contable, de manera que permita dar cuenta de su naturaleza interna y externa. Así mismo, apunta a dar a conocer cómo emerge el paradigma objetivo y subjetivo del sistema contable desde las perspectivas fundadas en la lógica formal clásica aristotélica, que ha perdurado hasta nuestros días, señalando un devenir de carácter causalista con arreglo a fines/medios para satisfacer y salvaguardar el orden estructural, en que la estabilidad y la identidad son pilares fundamentales.

Es preciso anotar que la decisión metódica y metodológica de comprender el sistema contable a partir de un arreglo con acuerdo a fines enfrenta las concepciones que se han elaborado sobre sistema contable y sus resultados a un panorama polémico. Se trata de un panorama caracterizado por la presencia de variadas concepciones, que, en el plano de la regulación contable internacional, es posible agrupar, pero imposible de homogenizar, estandarizar y uniformizar. Como consecuencia, se perfila una mirada unilineal para resolver las discrepancias por la vía de la imposición, si se quiere asumir el reto de la internacionalización de la economía.

En paralelo, se tornan de suma urgencia sus precisiones conceptuales en términos de equivalencias funcionales y la forma como definirán nuevas taxonomías para, a partir de ello, proceder al planteamiento de desarrollos eficaces para los elementos inherentes a la contabilidad. Lo que se constituyó en el centro de gravedad de la propuesta de investigación base fundamental de este artículo se relaciona con un replanteamiento de las categorías sobre las que se han sustentado las investigaciones para categorizar los sistemas contables 
existentes. Estas categorías se ubican en el entorno externo y en el entorno interno del sistema mismo, organizadas bajo un esquema causal que inhibe los rendimientos epistemológicos y metodológicos de cualquier estudio en este sentido.

En esta dirección, es preciso aclarar que el presente trabajo se dividió en dos momentos. En un primer momento, se realizó la selección y la sistematización de los enfoques teóricos que dan cuenta de los paradigmas objetivo y subjetivo que sobre sistema contable ha elaborado la comunidad científica contable.

Tabla 1. Criterios de selección y sistematización

\begin{tabular}{|l|l|l|}
\hline Momentos & \multicolumn{1}{|c|}{ Selección } & \multicolumn{1}{|c|}{ Sistematización } \\
\hline Criterios & Pertinencia & Correspondencia \\
\hline Rasgos & $\begin{array}{l}\mathrm{H}(\mathrm{h} 1, \mathrm{~h} 2, \mathrm{~h} 3 \ldots \mathrm{hn}) \in \mathrm{P} \\
(\mathrm{p} 1, \mathrm{p} 2, \mathrm{p} 3, \ldots \mathrm{pn})\end{array}$ & $\begin{array}{l}\mathrm{H} \subset(\mathrm{p} 1, \mathrm{p} 2, \\
\mathrm{p} 3 \ldots \mathrm{pn})\end{array}$ \\
\hline
\end{tabular}

Fuente: Elaboración propia, basada en Betancur y Cano (2018).

Con el criterio de selección, se describieron los contenidos de cada paradigma. En ese marco, «H» es el conjunto de hechos presentes en cada paradigma, mientras que «P» significa el conjunto de paradigmas que presentan el concepto de sistema contable. Entre ambos conjuntos, existe algún tipo de relación en la cual cada «P» describe y explica cada «H». Esta fase de selección pretende hacer una descripción de cómo un elemento de $\mathrm{H}$ pertenece a un elemento de P. La sistematización permitió establecer la correspondencia empírica o sector observacional que cada paradigma exhibe.

En un segundo momento, se presenta la esquematización, contenida de unos bosquejos en el orden ontológico y epistemológico, seguidos de una semiformalización bajo la forma $\mathrm{p} 1 \leftrightarrow \mathrm{pn}$. Este momento concluye con las definiciones y conceptos clave que se han levantado sobre sistema contable, como lo indica la Tabla 2.
Tabla 2. Criterio de esquematización

\begin{tabular}{|l|l|}
\hline \multicolumn{1}{|c|}{ Momentos } & \multicolumn{1}{c|}{ Esquematización } \\
\hline Criterios & Formulación esquemática \\
\hline Rasgos & p1 $\leftrightarrow$ pn \\
\hline
\end{tabular}

Fuente: Elaboración propia, basada en Betancur y Cano (2018).

En esta sección, se apuntará a iniciar un debate entre los diferentes paradigmas con respecto de la noción de sistema. Para ello, se necesita un saber de segundo orden, un saber que dé cuenta de otro saber, una ciencia de la ciencia, un estudio en el plano meta científico. Justamente, uno de ellos es la filosofía de la ciencia, la que despliega los estudios sobre la ciencia para entenderla.

Lo esbozado anteriormente significó hacer el tránsito por algunos de los conceptos fundamentales de estos pensadores. Esto sugirió un ejercicio interteórico en el que se debe entender que cada teoría de las diversas disciplinas científicas se halla en relaciones más o menos estrechas, y de diversa índole con otras teorías, con frecuencia de la misma disciplina, pero a veces también de disciplinas bastante distintas (Diez y Moulines, 1997, p. 367). También, permite observar la insuficiencia de la lógica interna de las teorías, las metodologías empleadas y el basamento experiencial como únicos referentes para el crecimiento del conocimiento científico. Su permanente replanteo, así como la consideración de otras cuestiones de carácter social; político; e, incluso, cultural son determinantes en dicho crecimiento. Ahora, junto con el contexto de la justificación, debe habitar el contexto del descubrimiento.

En esta misma dirección, evidenciar las dinámicas de estos cuerpos de conocimiento también sugiere pensar en el progreso de ellas con alguna permanencia, lo que insinúa un análisis diacrónico. Este análisis consiste en una referencia total de su evolución, desde 
la cual se realiza una reconstrucción de cada una de las diferentes pausas o momentos que conforman los planteamientos de Popper a Laudan — como se señalan a continuación-, no sin antes aproximarnos al contexto problemático que motiva este trabajo.

\section{Contexto problemático}

Para explicar la realidad, el ser humano se vale de teorías y, para ello, utiliza conceptos, puntos de vista (Wittgenstein, 1980, p. 18) racionalmente elaborados y demostrados, con los cuales se organiza el mundo. Estas teorías, a su vez, se pueden convertir en objetos de estudio, sobre los cuales se elaboran nuevos constructos más integrales para la observación del mundo. El presente trabajo pretende aproximarse a los discursos sobre una de las categorías fundamentales que utiliza la contabilidad: el sistema contable. A través de esta categoría, la comunidad pueda obtener información sobre el texto y contexto de lo contable.

Comprender la diversidad de concepciones que ha elaborado la comunidad contable sobre la noción sistema contable ayudará a entender las dificultades en el orden de la disciplina, la profesión, lo económico y lo organizacional (Betancur, 2017). Desde la dimensión disciplinar, los conceptos de los cuales se sirve la contabilidad se han vuelto disímiles, lo que ha conducido a la ambigüedad de marcos conceptuales para los usuarios de la información. Desde la dimensión profesional, los inconvenientes se han traducido en diversidad de prácticas relacionadas con el reconocimiento de los elementos contenidos en los estados financieros, su medición, preparación y presentación de información (Jarne, 1997). Diversidad y falta de uniformidad — con incidencia también en las formas de llevar a cabo la auditoría y control- en la aplicación de principios como esencia y forma han tenido repercusiones desde el punto de vista económico y fis- cal, así como en el nivel de formación profesional de los contables.

Desde la dimensión económica, se observan efectos para el sistema financiero como motor apalancador de la dinámica económica mundial (Salter, 1991). La diversidad de concepciones sobre sistema contable ha producido asimetrías de información que se derivan en distorsiones en el análisis e interpretación de la información, con sus respectivas repercusiones para la actividad inversionista en el plano internacional. Finalmente, a nivel de organización, se ha producido un incremento en los costos de la elaboración y la interpretación de la información contable financiera, que se traducen en costos de oportunidad y de pérdida de competitividad en el plano internacional del sector empresarial (Morales y Jarne, 2006).

El presente trabajo sostiene que la perspectiva objetiva y subjetiva que han fundamentado la concepción que la comunidad contable ha elaborado sobre sistema contable, antes que ser contradictorias y excluyentes, son complementarias y equivalentes funcionales que coadyuvan para un mejor entendimiento y comprensión de la realidad de la cual da cuenta la contabilidad (Rivera, 2011; Ivanovich, Peña y Torres, 2012). Sobre la base del anterior preámbulo, se plantea la siguiente pregunta que orientará este trabajo: ¿Cuáles son las concepciones que la comunidad contable ha elaborado sobre la categoría sistema contable que permiten ganar en información y hacer posible su reconstrucción?

\section{Paradigmas del constructo sistema contable}

Los conceptos y construcciones teóricas sobre los paradigmas (Kuhn, 1971), programas de investigación (Lakatos, 1983), el relativismo (Feyerabend, 1986) y las tradiciones de investigación (Laudan, 1986) se tradujeron en un itinerario que, desde la filosofía 
de la ciencia, se instaló a comienzos del siglo XX para favorecer la posibilidad de observar campos disciplinares, y comprender sus relaciones y contradicciones. En esta misma dirección, es posible hacer referencia a la evolución de la construcción y la reconstrucción del conocimiento científico. Para Kuhn (1971), por ejemplo, en la construcción de conocimiento, se da una fase preparadigmática y una fase posparadigmática. En esta última, se consolida el paradigma dominante, pero, antes de que esto suceda, hay concurrencia de múltiples paradigmas en competencia por el dominio del campo.

La contabilidad no se extrae de la dinámica anterior, como tampoco lo hacen algunas de sus categorías y conceptos más importantes. Así pues, el concepto de sistema contable está observado desde múltiples perspectivas que pueden considerarse paradigmas en competencia, debido a que cada uno de ellos reúne los presupuestos fundamentales contenidos en el paradigma, como lo son comunidad, consenso y un corte/comprensión de realidad. En esta dirección, los constructos que la comunidad contable ha elaborado sobre sistema contable se hallan en competencia, lo que sugiere su entendimiento y comprensión para poder lidiar con escenarios problemáticos de carácter interno - la disciplina y la profesión - y externo - la organización y el ámbito financiero-.

Desde el punto de vista interno, esta competencia de nociones sobre sistema contable ha llevado a que muchos de sus conceptos sean disímiles. A partir de ello, cualquier intento de un marco conceptual único, centrado en unos objetivos y unas prácticas comunes, se vuelve ambiguo, a lo que se suman los diversos intereses de la gran variedad de usuarios que hacen uso del concepto. En el campo profesional, tiene consecuencias en las prácticas relacionadas con el reconocimiento, medición, reparación y presentación de información, así como en las formas de llevar a cabo la auditoría y el control. La tensión entre conceptos como esencia y forma se traduce en sistemas contables diferentes, y tiene repercusiones en la fiscalidad en los determinados contextos donde estos se reproducen, lo cual, a su vez, tiene repercusiones en el nivel de formación profesional de los contables.

Desde el punto de vista externo, en la organización, la competencia de concepciones sobre sistema contable ha producido un incremento en los costos de elaboración e interpretación de la información. Asimismo, ha evidenciado las distorsiones informativas que se pueden derivar de dicha competencia, y que se pueden convertir en costos de oportunidad y de pérdida de competitividad en el plano internacional del sector empresarial. Finalmente, dicha competencia obliga a tener en cuenta un panorama multiparadigmático del sistema contable. Lo anterior tiene efectos para el sistema financiero como motor apalancador de la dinámica económica mundial. Las asimetrías de información derivadas de dicha uniformidad/diversidad producen distorsiones en el análisis e interpretación de la información, lo que repercute en la actividad inversionista en el plano internacional, al no contar con un sistema de información claro y uniforme.

El presente trabajo propone dar cuenta de dicho evento multiparadigmático del constructo sistema contable para que, desde ese punto, se allanen otras vías encaminadas a la construcción y reconstrucción teórica de la contabilidad.

\subsection{Paradigma objetivo}

El paradigma objetivo puede ser considerado como la corriente principal y la que contempla mayores desarrollos sobre sistema contable. Su referente es Richard Mattessich (1956, 1957), quien argumenta que la contabilidad moderna inicia su recorrido cuando la 
teoría general de la contabilidad se pone a prueba en los sistemas contables, momento en el cual florece la investigación normativa en contabilidad

\section{Selección}

Los inicios del siglo XX se caracterizan por la irrupción de eventos de carácter social, político y económico que delinearían su devenir. De este modo, en la primera mitad de dicho siglo, emerge la necesidad de una lógica postulacional para la contabilidad y el sistema contable. Representantes de este primer impulso fueron Aukrust, Chambers, Ijiri y Moonitz, según Mattessich (1972). Autores como Patón, Canning y Gilman se dedicaron a la tarea de revisar las incoherencias del cuerpo teórico contable, de acuerdo a Chambers (1980).

Mattessich postula la axiomatización para encaminar lo contable $(1956,1957)$. Dicha óptica supone una distinción entre unas tesis iniciales y otras derivables por implicación lógica, que conforman una estructura de carácter deductivo. En los años 70, el autor advertía que «el núcleo del problema podría descansar en la dificultad de formular objetivos específicos bien definidos y equipararlos a un conjunto específico de hipótesis» (Mattessich, 1972, pp. 478-479), como el camino que hay que destrabar para lograr tal unificación.

En esta dirección, propone la posibilidad de la integración de la contabilidad con una teoría general de la contabilidad, a través de la construcción de una estructura teórica puesta en evidencia cada vez que un sistema contable específico se pone a prueba. «La comprobación (verificación o refutación) de una teoría general de la contabilidad debe estar invariablemente ligada a las proposiciones empíricas específicas que rigen los sistemas de contabilidad particulares para su uso en la práctica real» (Mattessich, 1972, p. 485).
En el sistema contable, se cumple esta lógica deductiva (Mattessich, 1995a, p. 259).

Para Mattessich (2012), la contabilidad es una ciencia empírica y aplicada. Por un lado, es empírica, en tanto sus enunciados pueden ser contrastados en la experiencia, es decir, en los sistemas contables, cuando estos logran o no los propósitos previstos. Por otro lado, es aplicada, porque cada sistema contable demanda hipótesis desde las cuales se elaboran reglas en función de objetivos.

\section{Sistematización}

Los objetivos se presentan como el campo de observación, el constructo alrededor del cual gira este enfoque de carácter hipotético-deductivo. No importa tanto si la norma contable está generalizada y legitimada, o si se deriva de contenidos lógico formales. Lo importante es que la norma contable se supedite a objetivos, de tal manera que, con los objetivos como fundamento, la norma se tradujo en un medio para la consecución de un fin, argumentos centrales de la propuesta condicionalnormativa (Mattessich, 1995b). Este evento le adjudica el título de ser una disciplina utilitarista, aspectos que le son propios a la contabilidad y con los que deberá lidiar.

Frente a la imposibilidad de que la disciplina contable prescinda de la subjetividad de los objetivos, Mattessich la incorpora conscientemente a su epistemología. Lo que acontece en medio de la relación fines/medios (las hipótesis instrumentales) provee el conocimiento nomológico que permitirá el establecimiento de objetivos claros, evento que liberaría del sesgo subjetivista a la disciplina y haría coincidir la representación con el propósito.

Al enfoque hipotético-deductivo de Mattessich sobre el sistema contable, se adjuntan otras vertientes complementarias a este, puesto que comparten su perspectiva 
y sobre todo su campo empírico de observación, así como su basamento onto-epistemológico. Tales son los casos de los enfoques normativo-positivo, positivo, descriptivo-normativo, neoinstitucional y semántico, que se desarrollan a continuación.

\subsubsection{Vertiente positiva-normativa}

Desde esta orientación, Tua Pereda (1995) refrenda la postura deductiva postulacional y semántica para la construcción de la teoría general de la contabilidad y del sistema contable, observando como referente la evolución de los principios contables con los que ha operado el sistema contable norteamericano.

\section{Selección}

Según Tua Pereda (1995), mientras no se establezca la distinción entre la teoría general de la contabilidad (en adelante, TGC) de sus aplicaciones (sistemas contables), difícilmente se avanzará en la dimensión epistemológica y metodológica de la contabilidad, y los consecuentes inconvenientes para los procesos de regulación contable internacional. En este sentido, el autor afirma: «En cualquier caso, la más elemental distinción que pueda establecer el conocimiento contable es la diferencia entre sistema contable y teoría general de la contabilidad» (Tua Pereda, 1995, p. 339).

Este autor afirma que, para cada sistema contable, existe un conjunto de reglas diferentes que operan a partir de un conjunto de objetivos de acuerdo con unos entornos particulares, elementos que son de carácter normativo y teleológico con fuertes componentes de juicios de valor. Ello permite colegir el hecho de estar en presencia de sistemas contables en competencia, a partir de lo cual se evidencia que los obstáculos a superar para que la contabilidad alcance su estatus disciplinal se encuentran a nivel del sistema contable.

\section{Sistematización}

En el seno de las dinámicas socioeconómicas de la segunda mitad del siglo XX, emergen usuarios particulares (stakeholders) con ciertas necesidades de información útil para tomar decisiones. En este contexto, se declara de manera definitiva a los objetivos como el faro orientador, fines que debían satisfacerse con un conjunto adecuado de reglas. Así, el referente teleológico para la disciplina y el sistema contable se consolidan; ahora, el centro de interés son los objetivos. Su incidencia se empieza a evidenciar no solo en el desarrollo disciplinar, sino en la regulación llevada a cabo por organismos internacionales.

\subsubsection{Vertiente positiva}

Los albores de 1970 fueron fundamentales para esta teoría. Los cambios en el orden social, económico, político y cultural facilitaron su desarrollo. El auge de la socioepistemología, el progreso de ciencias — como la economía o las matemáticas-y los desarrollos de la teoría de la información, y la emergencia del paradigma científico-tecnológico propiciaron un ambiente favorable para realizar la investigación empírica.

\section{Selección}

Según Watts y Zimmerman (1990), la teoría positiva moderna empezó a darse entre los años 60 y 70 con los trabajos de Beaver (1968), y de Ball y Brown (1968). Esta primera etapa, a pesar de que dio inicio a la relación entre la información contable y el mercado de capitales, en su momento, no proporcionó hipótesis que posibilitaran la predicción y la explicación en esta economía. Esto se debió a que no se tuvo en cuenta que la información contable no es gratuita, lo que supone que hay costos de transacción que afectan el valor de la empresa (Watts y Zimmerman, 1990, pp. 132-133). 
De acuerdo con Watts y Zimmerman (1990), el aporte más importante de esta teoría es la puesta en escena de una teoría de la contabilidad y del sistema contable, pensada sistemáticamente y desde la cual se proponen normas contables en función del papel que desempeñan los costos contractuales. La teoría contable y el sistema contable hacen parte de una estructura organizacional de contratación eficiente, cuyo objetivo es la maximización del valor empresarial. De esta forma, la norma contable y los usuarios de la información se convierten en objeto económico y político.

\section{Sistematización}

Plan de compensaciones, endeudamiento, tamaño de la empresa, inflación y los fenómenos de la economía financiarizada en general son ejemplos de los nuevos eventos que ampliaron las perspectivas acerca de lo contable. Mouck (1990) considera que este replanteo para la teoría positiva es progresivo, puesto que se predicen y explican nuevos hechos que hasta ese momento no se habían tenido en cuenta.

Puede entenderse la teoría positiva de la contabilidad y del sistema contable como un corpus ordenado de conocimientos que da cuenta de lo que es. La teoría contable positiva, según Watts y Zimmerman, predice con el objetivo de explicar los diferentes eventos socioeconómicos observados, con lo que preparan el camino del enfoque normativo y la factibilidad de su orientación prescriptiva.

\subsubsection{Vertiente descriptiva-normativa}

En la segunda mitad del siglo XX, la internacionalización de los mercados se hizo intensa, al mismo tiempo que demandó de información económico-financiera homogénea. En este contexto, se inició un proceso de regulación contable buscando la uniformidad de la información, a la vez que se iniciaron los trabajos de investigación encaminados a verificar dicha homogenización, escenario que dio nacimiento a los estudios preliminares de la contabilidad internacional (Mueller, 1967) dando paso a investigaciones para caracterizar los sistemas contables existentes.

\section{Selección}

En la década de los 80, Nair y Frank (1980, 1982), a partir de bases de datos suministradas por multinacionales de auditoría, llevaron a cabo un estudio comparativo a nivel internacional de prácticas contables. Después de observar 267 prácticas contables en 64 países, concluyeron que las prácticas de divulgación son menos estables que las de medición, lo que explica la mayor diversidad de las primeras. A partir de estos trabajos, realizaron una clasificación de sistemas contables en cuatro grupos: el británico, el latinoamericano, el continental europeo y el estadounidense. Estos trabajos brindaron la posibilidad de aislar aquellas prácticas contables causantes de las diferencias entre sistemas contables y tratarlas de manera particular, elaborando políticas contables. Esta tarea, desde entonces, no ha sido fácil y, según estos autores, debe iniciarse a partir de la reducción de la diversidad de las prácticas de divulgación, debido a que es con respecto a estas que existe mayor dispersión. Dicha labor se hace más difícil si no se atiende la complejidad que se deriva de la observancia de las variables de entorno económico y cultural de cada país.

De acuerdo con Nobes (1991), las asimetrías producidas por la economía globalizada han generado que los entornos se vuelvan diferentes, cuestión que deriva en una heterogeneidad de sistemas contables. Según el autor, son seis las causas que motivan las diferencias a nivel internacional de los sistemas contables: entorno y cultura, aspectos legales, fuentes de financiación, sistema impositivo, nivel profesional e inflación. 
Nobes contrastó dos conjuntos de criterios que delimitan los sistemas contables. El primer conjunto de criterios abarca las variables explicativas (usuarios de la información, nivel de detalle de la norma contable y valor de la norma fiscal); el segundo grupo, las variables pragmáticas (conservadurismo, costo histórico, costes de remplazo, consolidación, provisiones/ reservas y uniformidad). Estos criterios fueron valorados de acuerdo con las estructuras definidas a partir de muestras realizadas para países desarrollados, que determinaron dos grupos: de base micro y macro uniforme (Nobes, 1983, p. 271). Los primeros fueron muy influenciados por la actividad económica (anglo); y los segundos, afectados por el gobierno y aspectos fiscales (continental europeo).

Jarne (1997), por otra parte, planteó la importancia de tener en cuenta en la clasificación de los sistemas contables todos los componentes que los integran, marco en que las prácticas contables es un componente más entre otros. El autor plantea que deben considerarse otros factores desencadenantes de las diferencias entre sistemas contables. Estos son divididos en causas externas (sistema legal, proveedores de financiación, nacionalismos, organización empresarial mercado bursátil, cultura y política) y causas internas (fiscalidad, usuarios, organización profesional, educación y objetivos de la contabilidad).

\section{Sistematización}

A partir de la clasificación de sistemas contables, sobre la base de las prácticas contables de divulgación (Nair y Frank, 1980-1982) —a lo que se suma el análisis de variables de entorno (Nobes, 1991)_, se empieza a concebir el sistema contable como un «todo» constituido de partes en continua interacción (Jarne, 1997). Jarne argumenta que, con antelación a cualquier caracterización o clasificación de sistemas contables, hay que proponer un esquema conceptual de estos, es decir, establecer los a priori que permitirían evaluar las reacciones del sistema frente a cambios y posibilitar su modelación previa. Este modelo se constituye en «un deber ser», integrado por aspectos funcionales llevados a cabo por las interacciones dadas entre subsistemas que, bajo una fuerza holística, propician el logro de objetivos.

\subsubsection{Vertiente neoinstitucional}

En este entorno de globalización económica y financiera del siglo XX, se empieza a consolidar primero un marco institucional (DiMaggio y Powell, 1999) y, posteriormente, uno neoinstitucional (North, 1990), los que posibilitarían la racionalidad económica y un supuesto orden social. Lo contable no se extrae de dicha dinámica. En ese contexto, empiezan a surgir instituciones encargadas de la regulación contable y del sistema contable a nivel internacional, proclives a esta racionalidad.

\section{Selección}

Con los acontecimientos derivados de la depresión de 1929, American Institute of Accountants ${ }^{1}$ (AIA), en asociación con New York Stock Exchange ${ }^{2}$ (NYSE), inicia la elaboración de declaraciones con gran incidencia en la contabilidad que empezaron a dar forma a los Principios de Contabilidad Generalmente Aceptados (GAAP, por sus siglas en inglés). De esta manera, comienza a desarrollarse la regulación contable internacional de naturaleza profesional, derivada de organismos estatales. Estos principios se convierten en guía para la elaboración de la información contable (Zeff, 1972; Cañibano, Tua y López, 1985;

\footnotetext{
1 En español, esta institución es conocida como el Instituto Americano de Contadores.

2 En español, es la Bolsa de Valores estadounidense.
} 
Tua, 1995). La regulación es resultado de la experiencia y de la práctica habitual como la mejor medida a seguir hasta hacerse obligatoria, avalada por la academia, instituciones de profesionales y gobiernos que le imprimieron legitimidad (Paton y Littleton, 1936; Sanders, Hatfield y Moore, 1938).

Dos décadas después, la urgencia de un camino más racional para la elaboración de normas contables (Moonitz, 1961; Sprouse y Moonitz, 1962), con los boletines ARS No. 1 y ARS No. 3 respectivamente, empezaron a constituirse en el preludio de la vía hipotético-deductiva que se demandaba para la construcción de reglas. A partir de ello, en la década de 1970, el ARB se convirtió en el Financial Accounting Standards Board (FASB), máximo organismo estadounidense emisor de regulación contable a nivel internacional, privilegio que empieza a compartirse con Europa a través de la International Accounting Standards Committe (IASC).

En medio del ambiente económico financiero que se originó en estos años y que se consolidó tres décadas después, apareció un nuevo tipo de agente (Álvarez y Medialdea, 2010, p. 177), con capacidad de incidir en las estructuras de propiedad y de control de las organizaciones a nivel planetario. Así, nació el IASB, una entidad de carácter corporativo con el propósito de lograr comportamientos homogéneos en la elaboración de información financiera, y de proporcionar a los usuarios y auditores un marco de criterios que les indique cómo elaborar la información financiera.

\section{Sistematización}

En el marco de los siglos XX y XXI, la contabilidad y el sistema contable dieron inicio a un panorama regulativo que puede entenderse desde tres momentos. El primero es la regulación contable internacional institucional-estatal, con el FASB americano como organismo regulador (siglos XIX-XX). En el segundo, desde el nivel neoinstitucional-interestatal, surge la hegemonía regulativa, que empieza a ser compartida entre el FASB y el IASC europeo (1970-1990). Finalmente, en el nivel corporativista, el IASB (desde el 2000 a la actualidad) inicia un proceso de convergencia con su antecesor ${ }^{3}$. Estas tres orillas dan cuenta de un igual número de transiciones. La primera fue el paso de las prácticas contables de uso generalizado, fruto de la experiencia y la experticia profesional hacia una segunda menos pragmática y más apuntalada en lo racional y en la lógica postulacional. En la tercera orilla, quedan aseguradas las bases de una taxonomía hipotético-deductiva, en la cual los objetivos se hacen determinantes, así como los estándares y los medios para lograrlos.

\subsubsection{Vertiente semántica}

La enseñanza-aprendizaje llevada a cabo en algunas universidades latinoamericanas ${ }^{4}$ sobre lo que es el sistema contable permitió inferir que lo transmitido es entendido como modelo contable, sistema de información, sistema administrativo contable, proceso contable y artefacto tecnológico.

\footnotetext{
3 Por requerimientos de la SEC (Securities and Exchange Commission), las compañías que cotizasen en alguno de los mercados financieros debían reconciliar sus cifras con la normativa norteamericana. A partir del 2002 y del 2005, el FASB norteamericano y el Cinif mexicano iniciarían programas de convergencia y adaptación respectivamente, con los IFRS del IASB. En el presente trabajo, convergencia hace alusión a la concurrencia hacia un mismo fin. Ese fin no es otro que el de las NIIF/IFRS y que se puede dar de dos maneras: adoptar (hacer como propia la normativa internacional sin discusión) y adaptar (hacer los ajustes que más convengan).

4 Esto depende del acercamiento realizado a algunos programas de estudio de algunas escuelas de contaduría pública colombianas y latinoamericanas (Betancur, 2017, p. 29).
} 


\section{Selección}

Es importante que, para un constructo sobre sistema contable, se exploren los sentidos asignados al concepto intentando aproximarlos. La siguiente es una mirada general a las anteriores concepciones, teniendo en cuenta que deben entenderse como complementarias, las que forman parte de un campo semántico.

- Modelo contable: La abstracción y la generalización de la teoría general de la contabilidad se hace manifiesta con modelos, los cuales representan describiendo y explicando la realidad de las organizaciones. Para hacerlo, estos se valen del contexto y de objetivos predispuestos por los usuarios de la información (García-Casella, 2001; Machado, 2016).

- Sistema de información: En la segunda mitad del siglo XX, se alzó con fuerza el desarrollo de la teoría de la información (Shannon, 2001, p. 6-7). Este campo surtió teórica y metodológicamente los elementos para desarrollar modelos generales de sistemas de información en contabilidad. Desde esta óptica, el sistema de información se resume en entrada, procesamiento, salida y retroalimentación.

- Sistema administrativo contable: El sistema administrativo contable puede entenderse como una entidad que coordina el conjunto de sistemas de información (contabilidad, finanzas, gestión, control, social, estratégico, costos, producción) que interactúan entre sí para apoyar las actividades de planeación, organización, dirección y control llevadas a cabo por una organización (Laudon y Laudon, 2012, p. 15).
- Proceso contable: Además de la teoría general de la contabilidad y su modelación a través del sistema contable, es necesario que los datos surgidos de las diferentes áreas funcionales se capturen, procesen y trasmitan de manera coherente. Su propósito consiste en registrar los hechos económicos conforme a los criterios de reconocimiento, medición y revelación propios de la información financiera útil (Contaduría General de la Nación, 2016, p. 4).

- Artefacto tecnológico: La conexión que se lleva a cabo a través del sistema contable entre la teoría y la práctica se ve fortalecida por lo tecnológico, no solo a nivel metodológico (procesamiento), sino a nivel de dispositivos (artefactos). Estos artefactos tecnológicos permiten ordenar, clasificar y procesar grandes volúmenes de información, convirtiéndose en una puesta en escena que garantiza el accionar en contexto del sistema contable.

\section{Sistematización}

Tanto la teoría general de la contabilidad como su modelación son referentes conceptuales y abstractos. Por ello, a la luz de García-Casella (2000, 2001 y 2002), los modelos contables hallan en los sistemas contables en uso la forma de hacerse evidentes. Estos se constituyen en la bisagra entre la dimensión conceptual/abstracta y la dimensión técnico/aplicada llevada a cabo a nivel macro ${ }^{5}$ o micro ${ }^{6}$. De esta forma, el sistema contable empieza a observarse como sistema de información, el cual carecería de sentido si no forman parte integral de todos los niveles de jerarquía y autoridad para planear y diseñar estrategias para la consecución de objetivos de las diferentes áreas funcionales (sistema administrativo contable). Es necesario

\footnotetext{
5 Puede referir a un grupo de países — sistema contable continental europeo (Jarne, 1997) — o a un segmento de la realidad — sistema contable financiero (García-Casella, 2002)-.

6 Refiere al marco empresarial específico (Machado, 2016).
} 
que la información que fluye por las anteriores áreas funcionales circule siguiendo un conjunto de pasos que observen una secuencia lógica, que se materializa a través de una plataforma tecnológica que facilita el procesamiento del inmenso flujo de datos y lo transforme en información útil.

\subsection{Paradigma subjetivo}

Este campo se fundamenta en teorías organizacionales, sociológicas y psicológicas, enfocadas en aspectos relacionados con la organización y las personas para tratar de establecer la incidencia de estos en la elaboración del sistema contable. De una manera distinta a la lógica postulacional, se abre un fructífero panorama teórico complementario. Tres de esas orientaciones teóricas son las de Roberts y Scapens (1985), Richard C. Laughlin (1984, 1987), y Anthony G. Hopwood (1987).

\section{Selección}

Roberts y Scapens (1985) encuentran que las prácticas contables son el camino para integrar los aspectos técnicos y conductuales en la organización, escenario por excelencia de la contabilidad y el lugar para el cual son pensados los sistemas contables. Para demostrar lo anterior, los autores tuvieron como referente a Anthony Giddens (1979, 1987), por considerarlo una postura integradora contemporánea. Los autores establecen la diferencia entre sistema contable y sistema de rendición de cuentas. Sobre el primero, argumentan que es un constructo dado a nivel abstracto; con el segundo, hacen referencia a los sistemas contables en uso. Hacer parte de una organización es estar condicionado funcionalmente por un sistema de rendición de cuentas (sistemas contables en uso); por otra parte, es la posibilidad de reproducirse en estos. Los sistemas contables en uso despliegan estructuras de significado contenidas de sentido, legitimación y poder, formas provistas desde el sistema contable y que son apropiadas por la contabilidad y la organización para ordenar sus experiencias y orientar su comportamiento (Roberts y Scapens, 1985, p. 448).

De su lado, Laughlin (1987) señala que, aunque es mucho lo que se sabe de los sistemas contables desde el punto de vista técnico, es poco lo que se sabe de ellos en la práctica. Si bien sus aspectos técnicos son ineludibles, estos deben entenderse en función del contexto en que se encuentren. Uno de esos escenarios es la organización. En estos lugares, adquieren significados más tangibles si se entienden en el sentido del lenguaje organizacional, en tanto el lenguaje se constituye en el elemento fundamental para producir la comprensión, el cambio y el desarrollo no violento, según Habermas (1981), pensador que le sirve de referente a este autor. De acuerdo con Laughlin, lo que debe preocupar

es nuestra incapacidad para diferenciar y retener como separadas las esferas sociales y técnicas ha llevado, según Habermas, a un proceso de "colonización interior» del mundo de la vida social por el sistema técnico. La esfera técnica domina lo social, superando ampliamente sus fronteras. (1987, p. 486)

En esta lógica, se apuesta por comprender las interrelaciones de lo social y lo técnico del sistema contable, pero además se propicia el cambio y su desarrollo. Para Laughlin, si Habermas está en lo correcto, el lenguaje ha producido la fractura entre lo técnico y lo social, y debe ser el mismo lenguaje el que debe permitir el acceso diferenciado de ambos. Es todo un proceso de descubrimiento desde una perspectiva crítica sobre la naturaleza fundamental de un sistema contable.

Es preciso mencionar que, desde la perspectiva de Hopwood (1987), el fenómeno de cambio contable no ha sido abordado suficientemente. Su referencia se ha realizado desde cuerpos de conocimiento externos a la contabilidad: la relación entre teoría contable y sistema 
contable ha sido un proceso emergente que ha derivado de procesos coyunturales. El autor sugiere que, en lugar de observar las prácticas contables dadas en el sistema contable de la organización como un reflejo de imperativos económicos, ahora deben ser vistas como un poderoso medio de gobierno y control organizacional. Una mirada de la contabilidad y del sistema contable desde este punto de vista trasciende lo técnico y se ubica en lo que fueron sus orígenes, un ejercicio de un poder social adentro y fuera de la organización, tal como se advierte en el siguiente argumento: «la contabilidad ha sido implicada en la transformación radical de la organización en nombre de lo social. Además, la contabilidad es considerada como uno de los más importantes medios por los cuales la organización se incorpora al dominio social» (Hopwood, 1987, p. 213). De este modo, el autor sugiere que la teoría contable y el sistema contable ya no solo es un oficio aislado adecuado a fines. Ante todo, es un coexistir, una interdependencia con la sociedad y la organización, articuladas desde las prácticas contables llevadas a cabo en el sistema contable.

\section{Sistematización}

Desde el enfoque subjetivo, Roberts y Scapens (1985) argumentan que la concepción técnica confundió las descripciones que las personas realizaron sobre el sistema contable con la forma en que estos se desenvuelven y conforman en la práctica. En esta dirección, los autores plantean la necesidad de integrar los aspectos técnicos de los sistemas contables con los conductuales. Las prácticas contables se constituyen como el camino para lograrlo y la teoría de la estructuración de Anthony Giddens es su soporte. En esta línea, Laughlin sostiene que los sistemas contables son más que elementos técnicos: están formados por fenómenos sociales y culturales; en ese sentido, cualquier cambio en estos sistemas implica que las raíces sociales y culturales también deben ser comprendidas y cambiadas. Su comprensión es posible desde el contexto organizacional y los lenguajes que de estos se derivan, códigos comunicativos que encuentra en la teoría crítica de Habermas su principal aliado teórico-metodológico. Por último y de manera complementaria, Hopwood indica que los verdaderos mecanismos de cambio se hallan en las prácticas contables en uso, poderosos artefactos de gobierno y control organizacional.

Son pocos los trabajos realizados desde la dimensión subjetiva. En esta, se empieza a comprender y entender la teoría contable a través del sistema contable al interior de las organizaciones. Se manifiesta, entonces, una suerte de dicotomía: de un lado, se entiende como una imagen y, de otro, como algo concreto con presencia real en las organizaciones, es decir, sistemas contables en uso y acción. A pesar de que ya se empieza a saber cómo el cambio contable favorecido por la adopción de nuevas prácticas en los sistemas contables transforma la organización, lo contable sigue observándose desde una óptica funcional, como una función habilitadora del desempeño organizacional, orientada en términos de necesidades y requerimientos organizacionales. Su papel es más el reflejo de ciertas circunstancias de contexto.

Lo que se debe empezar a trabajar también es el sistema contable en uso al interior de las organizaciones, como evento creativo, constructivo y transformador. Así, lo expresan las propuestas de Roberts y Scapens (la idea del sistema contable en uso a través del sistema de cuentas), Laughlin (lo cultural como fundamental para su crítica/cambio) y Hopwood (el sistema contable en acción a partir del papel constitutivo de las prácticas contables como factor de cambio). Desde esta perspectiva, el sistema contable es un estar en permanentemente en movimiento; su dinámica hace aparentemente real aquello que es abstracto.

Ello significa que la contabilidad y el sistema contable pueden lograr que ciertos eventos organizacionales 
lleguen a ser lo que no eran; producen, en ese sentido, el cambio externo, así como el propio. Hay una fuerza interna que mueve al sistema contable hacia la interacción e interdependencia organizacional, un aspecto constitutivo de la contabilidad aún por explorar. Estas son precisamente las condiciones de posibilidad desde las que empieza a producirse y reproducirse el entendimiento y la comprensión del sistema contable desde y para la organización.

\section{Esquematización}

Inicialmente, se presenta cómo la dimensión ontológica y epistemológica han servido de fundamento para que los paradigmas objetivo y subjetivo eleven su concepción de sistema contable (Tabla 3). Seguidamente, haciendo uso de un lenguaje semiformalizado (Bunge, 1988), se indica su formulación esquemática. Para finalizar, se muestra la noción y los conceptos claves sobre sistema contable que ha elaborado cada uno de estos dos paradigmas y las vertientes teóricas contenidas en estos (Tabla 4).

Tabla 3. Dimensiones ontológica y epistemológica de los paradigmas objetivo y subjetivo del sistema contable

\begin{tabular}{|l|l|l|}
\hline Dimensión & \multicolumn{1}{|c|}{ Ontología } & \multicolumn{1}{c|}{ Epistemología } \\
\hline Objetiva & $\begin{array}{l}\text { Realidad objetiva } \\
\text {-Objetiva- }\end{array}$ & $\begin{array}{l}\text { Constructos representando } \\
\text {-Objetiva- }\end{array}$ \\
\hline Subjetiva & $\begin{array}{l}\text { Negación de la rea- } \\
\text { lidad objetiva } \\
\text {-Subjetiva- }\end{array}$ & $\begin{array}{l}\text { Negación de constructos } \\
\text { representando la realidad } \\
\text {-Subjetiva- }\end{array}$ \\
\hline
\end{tabular}

Fuente: Elaboración propia, basada en Betancur y Cano (2018).

La dimensión objetiva se enmarca en lo que podemos considerar la investigación tradicional y dominante del sistema contable. En el plano ontológico, los objetos que se sitúan en el sistema contable poseen un correlato real; es decir, ostentan una existencia objetiva.
Epistemológicamente, desde el referente teleológico, esta dimensión propone unas hipótesis instrumentales asentadas en una relación causa-efecto. Estas permiten la apropiación de ciertas regularidades (nomológicas) que son aplicadas a una relación medios-fines, dirigidas a lograr una representación orientada por objetivos. En la dimensión subjetiva, los sistemas contables en uso, lo cultural como fundamento para la crítica y las prácticas contables como factor de cambio se constituyen en un deber ser que es una clara manifestación ontológica de carácter subjetiva. Epistemológicamente, esta dimensión se enmarca en un plano racional que plantea, desde lo que es, lo que podría ser de otra forma, un itinerario en la dimensión epistemológica de lo subjetivo.

En la dimensión objetiva del sistema contable desde Mattessich (1995a), las hipótesis instrumentales se traducen en enunciados prescriptivos de la forma: para lograr el objetivo A, dadas las condiciones $\mathrm{C}$, haga $\mathrm{D}$. Son las prescripciones propias de una ciencia aplicada, en la que la relación fines $\mathrm{A}$ y medios $\mathrm{D}$ es una determinación eminentemente teleológica y que toma la forma $\mathrm{A} \rightarrow \mathrm{D}$. Con la perspectiva positiva-normativa de Tua Pereda (1995), se reafirma la distinción entre prescriptivo y descriptivo. En el primero, lo importante es tener en cuenta los medios más adecuados para lograr un fin, mientras que el segundo permite los contrastes entre la realidad y el logro de objetivos pragmáticos.

Con la vertiente positiva, se explican las razones por las cuales los usuarios seleccionan determinadas normas contables, elección que viene dada no por ser la mejor medición de un evento contable; más bien, su utilidad radica en la resolución de ciertos problemas. Esta orientación adopta una perspectiva descriptiva (explica-predice) de la contabilidad y del sistema contable, con lo que se establece una relación 
de causa-efecto entre un fin y los medios. De esta manera, los enunciados de la teoría positiva adoptan la forma «Si A, dadas las condiciones C; entonces, D». Es una explicación acerca de una relación o determinación causal, por lo cual, cada vez que suceda A, también se producirá $\mathrm{D}$, siempre que estén dadas las condiciones C. Es decir, A sería la causa de D.

La vertiente descriptivo-normativa que inicia con Nair y Frank (1980), seguida por Nobes (1991), apela a una visión inductiva desde la cual se realiza la clasificación de sistemas contables. De su lado, Jarne (1997) objetaría que, si bien la clasificación por vía inductiva es relevante, lo importante era reflexionar en el sistema contable por vía deductiva para su adecuada clasificación y caracterización. En esta dirección, era vital comprender las interrelaciones entre los componentes internos y externos para entender el sistema contable como un todo, una suerte de integralidad que se explica de la siguiente manera:

$A=$ Es una expresión.

$\mathrm{X}=$ Es una variable.

$\mathrm{A}=$ «Todos los sistemas contables son un conjunto de componentes en interacción, con lo que se produce una totalidad». Si A es verdadero, lo será para todos los valores de X.

ScX = Sistema contable

CnX= Componentes del sistema contable

$\mathrm{n}=$ Conjunto de componentes donde las prácticas contables es uno de ellos

$\mathrm{T}=$ Totalidad

$(\forall \mathrm{x})=$ Cuantificador universal que se lee «para todo»

Determinando el alcance de dicha expresión, se plantea la siguiente operación:

$\begin{array}{ll}\text { ScX } & \text { X es un sistema contable } \\ \text { CnX } & \text { X conjunto de componentes en interacción }\end{array}$
Si X es un sistema contable, entonces, es un conjunto de componentes en interacción y aplica para todo $\mathrm{X} y$ se representa $(\forall \mathrm{x})$ ScX $\rightarrow \mathrm{Cn}(\mathrm{X})$. Dado lo anterior, «si y solo si» es causa de T. De esta manera, se representa en la siguiente expresión:

$$
[\forall \mathrm{xx}] \mathrm{ScX} \rightarrow \mathrm{Cn}(\mathrm{X})] \leftrightarrow \mathrm{T}
$$

Por otra parte, la vertiente neoinstitucional explica cómo el marco conceptual del IASB 2010 vigente sugiere que la información tiene como objetivo básico ser «útil» para tomar decisiones por parte de un inversor. Esto significa que las normas contables emitidas bajo unos principios deben satisfacer este objetivo y las instituciones encargadas de su emisión deben velar por que se garanticen estos presupuestos. El que una información sea útil depende de si lo es para un inversor, prestamista o acreedor financiero, lo que se puede representar como $\mathrm{f}(\mathrm{x})$. En esta fórmula, «f» representa a información útil; $\mathrm{y}$ « $\mathrm{X} » \mathrm{al}$ inversor, prestamista o acreedor financiero.

Ello ocurre siempre y cuando «A, dadas las condiciones $\mathrm{C}$, entonces $\mathrm{D}$ » sea una explicación acerca de una relación o determinación causal, por lo cual, cada vez que suceda $A$, también se producirá «D», siempre que estén dadas las condiciones C. Es decir, A sería la causa de D, de lo que se deduce la expresión $\mathrm{f}(\mathrm{x}) \leftrightarrow(\mathrm{A} \leftrightarrow \mathrm{D})$.

Por último, en la vertiente semántica, se articulan las diferentes concepciones que se han levantado sobre el sistema contable. En esta vertiente, se puede identificar una perspectiva sistémica contenida de los componentes conceptual y material en constante interacción. Sin embargo, a la luz del presente paradigma, este se ha observado desde la segunda dimensión y, a partir de cualquiera de ellos, se infiere el sistema contable:

$\mathrm{p}=$ Modelo contable

$\mathrm{q}=$ Sistema de información

$\mathrm{r}=$ Sistema administrativo contable 
$s=$ Proceso contable

$\mathrm{t}=$ Artefacto tecnológico

Si bien cualquier proposición (p, q, r, s, t) es sistema contable, hacerlo solo a partir de uno de ellos es insuficiente para comprenderlo y entenderlo. De tal evento, deberá de complementarse dicha afirmación en el nivel operativo. De la sumatoria de hechos que dan cuenta del sistema contable descritos por las proposiciones (p, q, r, s, t), se infiere el Sc (sistema contable). A su vez, lo que se considere deberá de concebirse como p, q, r, s, t, simultáneamente. De esta manera, puede expresarse de la siguiente manera, en la que $(\mathrm{p} \wedge \mathrm{q} \wedge \mathrm{r} \wedge \mathrm{s} \wedge \mathrm{t}) \leftrightarrow$ Sc.

La dimensión subjetiva, por su parte, propone que las prácticas que se llevan a cabo en los sistemas contables en uso se constituyan como la unidad reflexiva desde la cual debe observarse el constructo. Estos argumentos pueden plantearse en el plano dialectico y, con ello, niegan a sus anteriores, hallando en Anthony Hopwood su síntesis. A continuación, se presenta el esquema para esta perspectiva:

$$
\{(\mathrm{A} \rightarrow \mathrm{D}) \wedge(\mathrm{A} \leftrightarrow \mathrm{D})\} \wedge \neg\{(\mathrm{A} \rightarrow \mathrm{D}) \wedge(\mathrm{A} \leftrightarrow \mathrm{D})\}
$$

La negación que antecede el segundo término se convierte en una ausencia, en la insuficiencia, en lo inconcluso de los presupuestos normativos y positivos para explicar la contabilidad y el sistema contable. Negar para nuevamente afirmar desde los argumentos de la teoría organizacional, cultural y conductual brinda la posibilidad de una mirada más integral del concepto. De esta manera, a lo eminentemente tradicional o dominante se suma una concepción sobre el sistema contable como evento creativo, constructivo y transformador.

Posteriormente, los pensadores vieron con claridad que, para comprender las cosas y su significado, se requería no solo conocer los elementos, sino, principalmente,

las relaciones entre los mismos. Por ello, fue necesario un nuevo enfoque debidamente estructurado para que, dado un objetivo determinado, se encuentren los caminos, los medios y las formas para alcanzarlo, tomando en consideración las posibles soluciones, de las que se elegiría la que dé mejores resultados, con máxima eficiencia y mínimo costo. Este nuevo enfoque es el de los sistemas.

Por su parte, el biólogo alemán Bertalanffy (1989) sostuvo que era necesaria una relación del pensamiento científico, que debía estar dada por un cambio en las categorías básicas del pensamiento, debido a que «De uno u otro modo estamos forzados a vérnoslas con complejidades, con 'totalidades' o 'sistemas', en todos los campos del conocimiento» (p. 3). Desde este pensamiento, Bertalanffy concibió la teoría de sistemas de una manera interdisciplinaria, capaz de trascender los problemas exclusivos de cada ciencia, y de proporcionar principios generales (sean físicos, biológicos, psicológicos, sociológicos, químicos, contables, financieros, económicos, entre otros) y modelos generales para todas las ciencias involucradas, de modo tal que los descubrimientos efectuados en cada ciencia pudieran utilizarse por las demás.

Esta teoría interdisciplinaria, denominada teoría general de los sistemas, demuestra el isomorfismo de las diversas ciencias, permite mayor aproximación entre sus fronteras y llena los espacios vacíos (espacios blancos) entre estas. Dicha teoría es esencialmente totalizante, en la medida que los sistemas no pueden ser comprendidos solo por el análisis separado y exclusivo de cada una de sus partes. Por este motivo, esta teoría se basa en la comprensión de la dependencia recíproca de todas las disciplinas y de la necesidad de su integración. A partir de allí, entendemos el porqué de los esfuerzos humanos encaminados a descubrir las relaciones existentes entre diversos fenómenos, con el fin de impedir o facilitar la aparición de ciertos fenómenos de este momento. 


\section{Por ello, invocando un poco la estulticia, es conveniente} poner especial cuidado y atención en la comprensión de los puntos que se abordarán a continuación, pues en ellos se basa fundamentalmente esta propuesta, de la contabilidad y del control interno con enfoque de sistemas, como la contacibernética.
Para finalizar esta sección sobre la esquematización de los paradigmas objetivo y subjetivo, a continuación, se presenta las definiciones y algunos conceptos, a partir de los cuales se ha elaborado la noción de sistema contable:

\section{Tabla 4. Noción y conceptos del sistema contable en los paradigmas objetivo y subjetivo}

\begin{tabular}{|c|c|c|c|}
\hline \multicolumn{2}{|c|}{ Paradigmas } & \multicolumn{2}{|c|}{ Definición y conceptos } \\
\hline \multirow[t]{6}{*}{ OBJETIVO } & $\begin{array}{l}\text { Hipotético/ } \\
\text { deductivo }\end{array}$ & \multicolumn{2}{|c|}{$\begin{array}{l}\text { "Mecanismo para la descripción cuantitativa de los flujos de renta y agregados de la riqueza» (Mattessich, } \\
\text { 1972, pp. 449-450). } \\
\text { Conceptos claves: Objetivos, usuarios, necesidades de información, toma de decisiones, requisitos, teoría } \\
\text { y metodología general de la contabilidad y del sistema contable, axiomatización, lógica deductiva e induc- } \\
\text { tiva, teleológica, fines/medios, hipótesis instrumentales, ciencia empírica y aplicada. }\end{array}$} \\
\hline & $\begin{array}{l}\text { Positivo- } \\
\text { normativo }\end{array}$ & \multicolumn{2}{|c|}{$\begin{array}{l}\text { "Un sistema contable es un conjunto de reglas orientadas a una finalidad concreta. Sistemas, subsistemas e, } \\
\text { incluso, subderivaciones de estos últimos constituyen la manera en que se manifiesta y aplica la disciplina» } \\
\text { (Tua Pereda, 1993, p. 98). } \\
\text { Conceptos claves: Deducción, entorno socioeconómico, postulado, normativo, objetivos, características, } \\
\text { requisitos, reglas concretas. }\end{array}$} \\
\hline & Positivo & \multicolumn{2}{|c|}{$\begin{array}{l}\text { "Conjunto de principios y prácticas que articula todo suministro de información financiera, cuyas espe- } \\
\text { cificaciones, relativas a objetivos, características y requisitos que deben reunir los datos que se publican, } \\
\text { se encuentran influenciados por los entornos en los que habitualmente opera y actúa la contabilidad» } \\
\text { (Rodríguez, 1993, p. 44). } \\
\text { Conceptos claves: Teoría positiva, investigación empírica, explicación, predicción, mercado de capitales, } \\
\text { costos de agencia, costos de contratación, programa de investigación positiva, teorías fundamentales, hipó- } \\
\text { tesis auxiliares, hipótesis testeables. }\end{array}$} \\
\hline & \multirow[t]{3}{*}{$\begin{array}{l}\text { Normativo/ } \\
\text { deductivo }\end{array}$} & $\begin{array}{l}\text { R. D. Nair } \\
\text { y Frank G. } \\
\text { Werner }\end{array}$ & $\begin{array}{l}\text { «Conjunto de principios, normas y prácticas que gobiernan el suministro de información } \\
\text { financiera en un momento del tiempo y en un lugar determinado» (Bernau, 1995, p. 994). } \\
\text { Conceptos claves: Prácticas contables de medición, divulgación, estudio comparativo, com- } \\
\text { ponentes cultural, social, económico, causal, descriptivo, clasificación. }\end{array}$ \\
\hline & & $\begin{array}{l}\text { Christopher } \\
\text { Nobes }\end{array}$ & $\begin{array}{l}\text { «Conjunto de principios y prácticas que articula todo suministro de información financiera, } \\
\text { cuyas especificaciones, relativas a objetivos, características y requisitos que deben reunir los } \\
\text { datos que se publican, se encuentran influenciadas por los entornos en las que habitualmente } \\
\text { opera y actúa la contabilidad», según Nobes (citado en Rodríguez Lago, 1993, p. 44). } \\
\text { Conceptos claves: Análisis, clasificación, interrelación racional, estructura deductiva, crite- } \\
\text { rios a priori, descripción, comparación, características básicas, grupo de sistemas contables, } \\
\text { variables financieras, fiscales, profesionales, inflación. }\end{array}$ \\
\hline & & $\begin{array}{l}\text { José Ignacio } \\
\text { Jarne J. }\end{array}$ & $\begin{array}{l}\text { "Conjunto de factores intrínsecos al propio sistema (agentes internos) que, a través de la } \\
\text { modelización de que son objeto por medio de sus propias interrelaciones y de las influen- } \\
\text { cias del exterior (agentes externos), conforman un 'todo' debidamente estructurado, capaz } \\
\text { de satisfacer las necesidades que a la función contable le son asignadas en los diferentes } \\
\text { ámbitos» (Jarne, 1997, p. 43) } \\
\text { Conceptos claves: Agentes internos y externos, subsistemas contables, componente, inte- } \\
\text { rrelaciones, "todo», estructura, variable necesidades, función recurrencia, iterativo, integra- } \\
\text { lidad, modelos anglo y continental }\end{array}$ \\
\hline
\end{tabular}




\begin{tabular}{|c|c|c|}
\hline \multicolumn{2}{|c|}{ Paradigmas } & Definición y conceptos \\
\hline & $\begin{array}{l}\text { Neo-insti- } \\
\text { tucional }\end{array}$ & $\begin{array}{l}\text { "Conjunto de variables del entorno social y de la dinámica interna de la contabilidad que tiene que ser } \\
\text { operacionalizada y representada por la contabilidad» (Gómez, 2011, p. 140). }\end{array}$ \\
\hline & & $\begin{array}{l}\text { Conceptos claves: Finanzas, institucional, neoinstitucional, corporativo, organización, estatal, interestatal, } \\
\text { regulación contable, SEC, IOSCO, FASB, IASB, Norwalk, principios, reglas, inversor, útil, acreedor, } \\
\text { aceptación generalizada, principios, gobierno corporativo, stakeholders, shareholders }\end{array}$ \\
\hline & Semántico & $\begin{array}{l}\text { «Conjunto de principios, normas y prácticas que gobiernan el suministro de información financiera, en } \\
\text { un ámbito espacial y temporal dado» (García, 1995, p. 55). }\end{array}$ \\
\hline & & $\begin{array}{l}\text { Conceptos claves: Sistema de información, abstracción, modelación, proceso, ciclo, información, fuente, } \\
\text { transmisor, canal, receptor, destinatario, administrativo, gerencial, áreas funcionales, usuario, útil, pro- } \\
\text { ceso, tecnología, entrada, procesamiento, salida, retroalimentación }\end{array}$ \\
\hline \multirow[t]{6}{*}{ SUBJETIVO } & $\begin{array}{l}\text { Roberts y } \\
\text { Scapens }\end{array}$ & $\begin{array}{l}\text { «Los sistemas no son estructuras, sino que los sistemas tienen estructuras, las cuales se producen y repro- } \\
\text { ducen solo cuando las personas interactúan unas con otras» (Roberts y Scapens, 1985, p. 446). }\end{array}$ \\
\hline & & $\begin{array}{l}\text { Conceptos claves: Sistema de rendición de cuentas, dualidad de la estructura, prácticas contables, sisté- } \\
\text { mico, estructura, operación, interacción, temporalidad, distancia, interacción }\end{array}$ \\
\hline & $\begin{array}{l}\text { Richard C. } \\
\text { Laughlin }\end{array}$ & $\begin{array}{l}\text { «Sistema formal basado en la empresa que se expresa en términos numéricos fundamentalmente acciones } \\
\text { financieras del pasado, presente y futuro de este tipo de empresas» (Laughlin, 1984, p. 8). }\end{array}$ \\
\hline & & $\begin{array}{l}\text { Conceptos claves: Elementos técnicos, raíces culturales, lenguaje, comunicación, teoría critica, Intersub- } \\
\text { jetivo, subjetivo, objetivo, cuasi-ignorancia, teoremas críticos, iluminación, estrategia, proceso discursivo, } \\
\text { consenso }\end{array}$ \\
\hline & $\begin{array}{l}\text { Anthony } \\
\text { Hopwood }\end{array}$ & $\begin{array}{l}\text { «El sistema contable es un todo complejo de prácticas contables reflejo de coyunturas organizacionales y } \\
\text { constitutivo del cambio organizacional, contable y de sí mismo, que propicia el diálogo continuo entre lo } \\
\text { posible y lo actual» (Hopwood, 1987). }\end{array}$ \\
\hline & & $\begin{array}{l}\text { Conceptos claves: Sistema contable en uso, cambio contable, cambio organizacional, proceso emergente, } \\
\text { prácticas contables, gobierno y control, coexistencia e interdependencia, movimiento, ojo contable, con- } \\
\text { trol social, contingencia, posibilidad }\end{array}$ \\
\hline
\end{tabular}

Fuente: Elaboración propia, basada en Betancur y Cano (2018).

\section{Conclusiones y recomendaciones}

Es oportuno destacar tanto el significado de la teoría general de los sistemas como las situaciones del pensamiento y el desarrollo de la ciencia que condujeron a la conceptualización de esta teoría. De una parte, la ciencia moderna se caracterizó por la especialización creciente, derivada de la gran cantidad de datos e información, aunada a la complejidad que había alcanzado la técnica y las estructuras teóricas de cada campo del conocimiento. De esta manera, la ciencia se escindió en innumerables disciplinas que permanentemente generaron nuevas subdisciplinas.
Cabe anotar, como en la revisión de los paradigmas del constructo sistema contable, que, en diversos campos distintos e independientes, surgieron problemas y concepciones similares para su solución. Con gran sabiduría, Bertalanffy indicó: «Este paralelismo de principios cognoscitivos generales en diferentes campos es aún más impresionante cuando se tiene en cuenta que se dieron independientemente, sin que casi nunca interviniera nada de la labor en indagación en campos aparte» (1989, p. 31).

En la primera mitad del siglo XX, aflora la edad de oro de la investigación a priori o normativa, que, 
con la lógica postulacional deductiva de Mattessich en la década de 1970, alcanza su máxima expresión y sirve de base para la investigación positiva en contabilidad. Esta orientación es la que surte a las instituciones encargadas de la regulación contable internacional de los elementos teórico-metodológicos para la elaboración de marcos conceptuales. Emerge, de esta forma, el paradigma de la utilidad de la información, en la cual convergen los conceptos de entorno, objetivos, usuarios, necesidades, información, decisiones y utilidad. Tal es el panorama actual de la contabilidad, caracterizado como el paradigma de la utilidad de la información.

Asimismo, se entiende que nada dictaba en aquellos tiempos ni en el presente cómo desembocar en sistemas tradicionales. Ya lo analiza la vertiente positiva-normativa, según la cual se deben buscar principios que sean aplicables a los sistemas en general. Si es adecuadamente planteado y definido el sistema, veremos que existen modelos, principios y leyes que se aplican a sistemas generalizados sin importar su particular género, elementos. Por ello, la teoría general de los sistemas no pretende dar una solución a todos los problemas que se presenten o intentar soluciones prácticas, sino elaborar una serie de teorías, formulaciones y declaraciones conceptuales que permitan.

Sin embargo, la vertiente positiva-normativa plantea la existencia de dificultades, en cuanto a la dimensión epistemológica y metodológica de la contabilidad en términos de los procesos de la regulación contable internacional. Dicha situación puede ser considerada a la luz del conocimiento de la teoría general de los sistemas, tal cual como la tomó la administración y acudiendo a sus principios fundamentales para entender a las organizaciones como sistemas abiertos. Es decir, importó estímulos del medio ambiente, representados por las entradas del sistema, para procesarlos adecuadamente y producir salidas. Tal escenario lleva a comprender la contabilidad como la fuente principal de la información del sistema y su contribución al desarrollo de esta vertiente.

Por otra parte, la vertiente positiva establece una serie de cambios en el orden social, económico, político y cultural que facilitaron su desarrollo, y dieron auge a la socioepistemología, el progreso de ciencias como la economía, las matemáticas, los desarrollos de la teoría de la información, dando posibilidades a que la contabilidad pueda lograr el empoderamiento de ciencia que tanto se discute en el medio académico.

Es mucho el camino que todavía falta por recorrer para lograr dar el estatus científico que merece la contabilidad, sin dejar de reconocer que autores como Mattessich, Bertalanffy, Jarne, Giddens, Tua Pereda, Laughlin, Roberts, Zimmerman y Scapens, entre otros, han realizado aportes significativos. Sus trabajos han desarrollado modelos y propuesto sistemas contables que han dado origen a los nuevos lineamientos mundiales sobre cómo la contabilidad y el manejo adecuado de las decisiones organizacionales vienen ganando espacios en el mundo científico, y tratan de interpretar la lógica semántica del quehacer conforme al deber ser de la contabilidad en el contexto mundial.

\section{Referencias bibliográficas}

Angulo, J. A. G. \& Garvey, A. M. (2015). El nuevo Marco Conceptual del IASB (propuesta de 2015): entre la filosofía y la componenda. AECA: Revista de la Asociación Española de Contabilidad y Administración de Empresas, (111), 34-39.

Álvarez, I. \& Medialdea, B. (2010). La influencia de la financiarización sobre el gobierno corporativo de la empresa: El papel de los inversores institucionales. Revista de Economía Mundial, 24, 165-191. 
Ball, R. \& Brown, P. (1968). An empirical evaluation of accounting income numbers. Journal of Accounting Research, 6(2), 159-178. https://doi.org/10.2307/2490232

Betancur, H. D. (2017). Elementos reflexivos para un análisis sistémico-constructivista de la noción «sistema contable». Revista Visión Contable, (15), 10-33. https://doi.org/10.24142/rvc.n15a1

Bunge, M (1988). La ciencia, su método y su filosofía. Buenos Aires: Siglo XX.

Cañibano, L., Pereda, J. T. \& López, J. L. (1985). Naturaleza y filosofía de los principios contables. Revista Española de Financiación y Contabilidad, 15(47), 293-355.

Chambers, R. J. (1980). The myths and the science of accounting. Accounting, Organizations and Society, 5(1), 167-180. https://doi.org/10.1016/03613682(80)90033-1

Contaduría General de la Nación (2016, 13 de setiembre). Resolución No. 525. Proceso contable y sistema documental contable. Recuperado de http://www.contaduria.gov.co

Díez-Alegría, J. M. \& Moulines, C. U. (1997). Fundamentos de filosofía de la ciencia. Barcelona: Editorial Ariel.

DiMaggio, P. \& Powell, W. (1999). El nuevo institucionalismo en el análisis organizacional. México: Fondo de Cultura Económica, Colegio Nacional de Ciencias Políticas y Administración Pública, UAEM.

Feyerabend, P. (1986). Tratado contra el método. Esquema de una teoría anarquista del conocimiento. Madrid: Editorial Technos.

García-Casella, C. L. (2000). Curso universitario de Introducción a la Teoría Contable. Primera parte. Buenos Aires: Editorial Economizarte.

García-Casella, C. L. (2002). El problema del uso de modelos en la contabilidad. Revista Internacional Legis de Contabilidad y Auditoría, 12, 199-235.

García-Casella, C. L. \& Rodríguez de Ramírez, M. (2001). Elementos para una teoría general de la contabilidad. Buenos Aires: La Ley.
Giddens, A. (1979). Central problems in social theory: Action, structure, and contradiction in social analysis (Vol. 241). Berkeley/ Los Angeles: University of California Press. https://doi.org/10.1007/978-1-349-16161-4

Giddens, A. (1987). El estructuralismo, el post estructuralismo y la producción de cultura en la teoría social hoy. Ciudad de México: Editorial Alianza.

Habermas, J. (1981). Teoría de la acción comunicativa. Madrid: Editorial Taurus.

Hopwood, A. G. (1987). The archaeology of accounting systems. Accounting, Organizations and Society, 12(3), 207-234. https://doi.org/10.1016/0361-3682 (87)90038-9

International Accounting Standards Board [IASB] (2001). Framework for the Preparation and Presentation of Financial Statements. London: IASB.

International Financial Reporting Standards Foundation [IFRS] (2013). Una guia para micro entidades que apliquen la NIIF para las PYMES (2009). Londres: IFRS Foundation Publications Department.

Ivanovich, J., Peña, A. \& Torres, I. (2012). Un análisis crítico a las NIIF-IFRS y a los procesos de adopción e implementación en América Latina y el Caribe. Macro proyecto de investigación. Recuperado de http://www.alafec.unam. $\mathrm{mx} /$ docs/macroproyectos/analisis_macro.pdf

Jarne, J (1997). Clasificación y evolución internacional de los sistemas contables. Madrid: AECA Monografías.

Kuhn, T. (1971 [1962]). La estructura de las revoluciones cientificas. Ciudad de México: Fondo de Cultura Económica.

Lakatos, I. (1983). La metodología de los programas de investigación cientifica. Madrid: Alianza Editorial.

Laudan, L. (1986). El progreso y sus problemas. Madrid: Editorial Encuentro.

Laudon, K. L. y Laudon J. L. (2012). Sistemas de información gerencial. Ciudad de México: Editorial Pearson Educación.

Contabilidad y Negocios (14) 27, 2019 / ISSN 1992-1896 
Laughlin, R. C. (1984). The design of accounting systems: A general theory with an empirical study of the Church of England (doctoral dissertation). University of Sheffield, Sheffield, Inglaterra.

Laughlin, R. C. (1987). Accounting systems in organisational contexts: A case for critical theory. Accounting, Organizations and Society, 12(5), 479-502. https://doi. org/10.1016/0361-3682(87)90032-8

Leibniz, G. W. (1983). Nuevos ensayos del entendimiento humano. Madrid: Editora Nacional.

Machado, M. (2016). Las creencias compartidas en el discurso modelo contable y sus consecuencias desde la perspectiva sociocognitiva y critica de Van Dijk (tesis doctoral). Universidad de los Andes, Mérida, Venezuela.

Mattessich, R. (1956). Hacia una fundamentación general y axiomática de la ciencia contable. Revista Técnica Económica, 4.

Mattessich, R. (1957). Towards a general and axiomatic foundation of accountancy. With an introduction to the matrix formulation of accounting systems. Accounting Research, 8(4). https://doi.org/10.1016/ 0361-3682(94)00023-O

Mattessich, R. (1972). Methodological preconditions and problems of a general theory of accounting. The Accounting Review, 47(3), 469-487.

Mattessich, R. (1995a). Conditional-normative accounting methodology: Incorporating value judgments and means-end relations of an applied science. Accounting, Organizations and Society, 20(4), 259-284.

Mattessich, R. (1995b). Critique of accounting: Examination of the foundations and normative structure of an applied discipline. Wesport: Quórum Books.

Mattessich, R. (2012). Instrumental reasoning and systems methodology: An epistemology of the applied and social sciences Dordrecht/Boston: Springer Science \& Business Media.
Moonitz, M. (1961). The basic postulates of accounting. Nueva York: American Institute of Certified Public Accountants, ARS del AICPA, No. 1.

Morales, F. y Jarne, J. I. (2006). Clasificación internacional de los sistemas contables de Argentina, Brasil y Chile. Panorama Socioeconómico, 24(32).

Mouck, T. (1990). Positive accounting theory as a Lakatosian research programme. Accounting and Business Research, 20(79), 231-239. https://doi.org/10.1080/0 0014788.1990 .9728881

Müller, G. (1967). International Accounting. The International Executive, 9(4), 1-2. Nueva York: Ediciones MacMillan. https://doi.org/10.1002/tie.5060090401

Nair, R. (1982). Empirical Guidelines for Comparing International Accounting Data. Journal of Accounting Business Studies, winter, 85-98. https://doi. org/10.1057/palgrave.jibs. 8490560

Nair, R. \& Frank, W. (1980). The impact of disclosure and measurement practices on international accounting classifications. The Accounting Review, 55(3), 426-450.

Nobes, C. (1983). A judgmental international classification of financial reporting practices. Journal of Business Finance and Accounting, 10(1), 1-20. https://doi. org/10.1111/j.1468-5957.1983.tb00409.x

Nobes, C. \& Parker, R. (1991). Introduction and causes of differences. Comparative International Accounting, 4, 3-21.

North, D. C. (1990). A transaction cost theory of politics. Journal of Theoretical Politics, 2(4), 355-367. https:// doi.org/10.1177/0951692890002004001

Paton, W. A. y Littleton, A. C. (1936). Tentative statement of accounting principles affecting corporate reports source. The Accounting Review, 11(2), 187-191.

Rivera, M. (2011). Representación contable: De la revelación de los hechos a la construcción de la realidad. Revista Lúmina, enero-diciembre (12), 152-171. Manizales: Universidad de Manizales. 
Roberts, J. \& Scapens, R. (1985). Accounting systems and systems of accountability—understanding accounting practices in their organisational contexts. Accounting, Organizations and Society, 10(4), 443-456. https://doi. org/10.1016/0361-3682(85)90005-4

Rodríguez, L., J. (1993). Tendencias contemporáneas en la clasificación de los sistemas contables. Técnica Contable, enero, 43-52

Salter, S. (1991). Classification of Financial Systems and Test of their environment al Determinants (doctoral dissertation). College of Business Administration, University of South Carolina, Carolina Del Sur, Estados Unidos.

Sanders T., Sanders, H., Hatfield, H. \& Moore, U. (1938). A statement of accounting principles. Nueva York: American Institute of Accountants.

Shannon, C. E. (2001). A mathematical theory of communication. ACM Sigmobile Mobile Computing and Communications Review, 5(1), 3-55. https:/doi. org/10.1145/584091.584093

Sprouse, R. T. \& Moonitz, M. (1962). A tentative set of broad accounting principles for business enterprises (No. 3). Nueva York: American Institute of CPAs.

Tua Pereda, J. (1995). Lecturas de teoría e investigación contable. Medellín: Centro Interamericano Jurídico-Financiero.
Tua Pereda, J. (2009). Contabilidad y desarrollo económico: El papel de los modelos contables de predicción especial referencia a las NIC'S. Ponencia presentada en el simposio Análisis y propuestas creativas ante los restos del nuevo entorno empresarial. Universidad ICESI y Revista Estudios Generales (pp. 1-28), Cali, Colombia.

Von Bertalanffy, L. (1989). Teoría general de los sistemas. Ciudad de México: Fondo de Cultura Económica.

Watts, R. L. \& Zimmerman, J. L. (1990). Positive accounting theory: A ten year perspective. The Accounting Review, 65(1), 131-156.

Wittgenstein, L. (1980). Culture and values. Chicago: University of Chicago Press.

Zeff, S. A. (1972). Chronology of significant developments in the establishment of accounting principles in the United States, 1926-1972. Journal of Accounting Research, 10(1), 217-227. https://doi.org/10.2307/2490230

Fecha de recepción: 12 de abril de 2019 Fecha de aceptación: 31 de mayo de 2019 Correspondencia: hdbetancur@udem.edu.co amcano@udem.edu.co 